\title{
Amodiaquine failure associated with erythrocytic glutathione in Plasmodium falciparum malaria
} Lina Zuluaga1, Adriana Pabón ${ }^{1}$, Carlos López ${ }^{2}$, Aleida Ochoa² and Silvia Blair*1

\author{
Address: ${ }^{1}$ Grupo Malaria, Universidad de Antioquia, Calle 62 \# 52-59, Torre 1, Piso 6. Laboratorio 610, Sede de Investigación Universitaria (SIU),

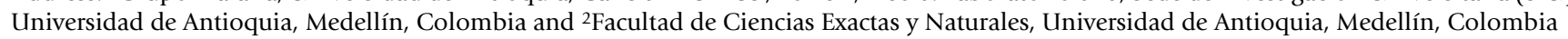 \\ Email: Lina Zuluaga - linazulu83@yahoo.es; Adriana Pabón - apabon@quimbaya.udea.edu.co; \\ Carlos López - carlopez@matematicas.udea.edu.co; Aleida Ochoa - aleida08@yahoo.com; Silvia Blair* - sblair@quimbaya.udea.edu.co \\ * Corresponding author
}

Published: 23 April 2007

Malaria Journal 2007, 6:47 doi:10.1186/1475-2875-6-47

This article is available from: http://www.malariajournal.com/content/6///47

(c) 2007 Zuluaga et al; licensee BioMed Central Ltd.

This is an Open Access article distributed under the terms of the Creative Commons Attribution License (http://creativecommons.org/licenses/by/2.0), which permits unrestricted use, distribution, and reproduction in any medium, provided the original work is properly cited.
Received: 23 November 2006

Accepted: 23 April 2007

\begin{abstract}
Objective: To establish the relationship between production of glutathione and the therapeutic response to amodiaquine (AQ) monotherapy in Plasmodium falciparum non-complicated malaria patients.

Methodology: Therapeutic response to AQ was evaluated in 32 patients with falciparum malaria in two townships of Antioquia, Colombia, and followed-up for 28 days. For every patient, total glutathione and enzymatic activity (glutathione reductase, GR, and $\gamma$-glutamylcysteine synthetase, $\gamma$ GCS) were determined in parasitized erythrocytes, non-infected erythrocytes and free parasites, on the starting day (day zero, before ingestion of AQ) and on the day of failure (in case of occurrence).
\end{abstract}

Results: There was found an AQ failure of $31.25 \%$. Independent of the therapeutic response, on the starting day and on the day of failure, lower total glutathione concentration and higher GR activities in parasitized erythrocytes were found, compared with non-infected erythrocytes $(p<$ $0.003)$. In addition, only on the day of failure, $\gamma$-GCS activity of parasitized erythrocytes was higher, compared with that of healthy erythrocytes $(p=0.01)$. Parasitized and non-parasitized erythrocytes in therapeutic failure patients (TF) had higher total glutathione on the starting day compared with those of adequate clinical response (ACR) $(p<0.02)$. Parasitized erythrocytes of TF patients showed lower total glutathione on the failure day, compared with starting day $(p=0.017)$. No differences was seen in the GR and $\gamma$-GCS activities by compartment, neither between the two therapeutic response groups nor between the two treatment days.

Conclusion: This study is a first approach to explaining $P$. falciparum therapeutic failure in humans through differences in glutathione metabolism in TF and ACR patients. These results suggest a role for glutathione in the therapeutic failure to antimalarials.

\section{Background}

Malaria is a parasitic disease, which has the highest mor- bidity/mortality rate in tropical countries [1]. The causal agent with the highest lethality rate for this disease and 
resistance to antimalarials in the world is Plasmodium falciparum [2]. In Colombia, there have been reports about resistance of $P$. falciparum to antimalarial drugs since 1960. In Antioquia, Colombia, $97 \%$ and $30 \%$ of therapeutic failure to the 4-aminoquinolines, chloroquine (CQ) and amodiaquine (AQ) respectively, have been reported $[3,4]$. Therefore, CQ is no longer used in Antioquia and the use of AQ in combination with sulphadoxine-pyrimethamine (SP) was suggested since 1985 and until 2006 [4].

Haemoglobin is degraded by Plasmodium during intraerythrocytic stages in a process where haem is released and converts itself into a toxic molecule, because this parasite lacks haem oxygenase and produces reactive oxygen species (ROS) [5]. The parasite detoxifies haem simultaneously by polymerization and degradation. In the first case, the parasite converts almost $30 \%$ of haem in haemozoin or malarial pigment. Haem toxicity is avoided too, through cytosolic degradation by reduced glutathione (GSH). This can degrade haem, whether it is free in solution or it is bound nonspecifically to protein, when dissolved in erythrocyte membranes or loaded into intact erythrocytes $[6,7]$ Plasmodium is endowed with a machinery to detoxify ROS. This antioxidant system comprises GSH and thioredoxin redox system, superoxide dismutase, NADPH and a vigorous pentose phosphate pathway, but it lacks catalase and glutathione peroxidase [810].

Glutahione is synthesized by the step-limiting enzyme $\gamma$ glutamyl cysteine synthetase ( $\gamma$-GCS) and by glutathione synthetase (GS). This thiol found in its reduced state inside all cells, contributes to maintaining intracellular redox state; besides, it has been suggested that GSH is involved in drug resistance both as a cofactor for enzymatic reactions and by helping to mediate resistance as a source of reductive detoxification of haem. After its reaction with a free radical, GSH changes its oxidation state (GSSG). Glutathione reductase (GR) NADPH is the enzyme responsible for keeping glutathione in its reduced state $[9,10]$.

The resistance of $P$. falciparum to the 4-aminoquinolines, such as CQ, has been shown to be associated with modification of drug transport by the membrane proteins Pglycoprotein homologue 1 (Pgh1) and the P. falciparum chloroquine resistance transporter (pfcrt) [10]. An additional mode of resistance has been suggested that is connected to an increase of GSH levels, which compete with the drug for degrading the haem toxin [7].

Meierjohann et al. [11] showed that growth inhibition of $P$. falciparum CQ-sensitive strain (3D7) by L-buthionine$(\mathrm{S}, \mathrm{R})$-sulfoximine (BSO), a specific inhibitor of $\gamma$-GCS and by methylene blue (MB), an inhibitor of the GR, was significantly more pronounced than inhibition of $P$. falciparum CQ-resistant strain (Dd2) growth by these drugs. These results correlate with the higher levels of total glutathione in $P$. falciparum Dd2. In addition, they suggest that maintenance of intracellular GSH in $P$. falciparum $\mathrm{Dd} 2$ is mainly dependent on GSH synthesis, whereas in $P$. falciparum 3D7 is regulated via GR [11].

Studies in vivo on Plasmodium berghei-infected mice confirm that drugs which alter GSH intracellular concentrations, change Plasmodium susceptibility to the CQ [12]. The correlation between GSH level and resistance CQ is supported by the higher expression of $\gamma$-GCS [13]. It has been shown that acquisition of CQ resistance in $P$. berghei is associated with a significant increase in parasite glucose 6-phosphate deshydrogenase (G6PD) activity and GSH. Combination of CQ with an inhibitor of G6PD or BSO significantly increased sensitivity of resistant parasites to CQ and increased the survival period of the infected mice [14]. Other studies in vivo on P. berghei and Plasmodium vinckei-infected mice showed that drugs, such as acetaminophen, indomethacin and disulphiram, which produce an indirect decrease in GSH, potentiate the antimalarial action of CQ and AQ sub-curative doses [15].

This study examined whether there exists a relationship between the total glutathione level in patients with malaria by $P$. falciparum and therapeutic failure to AQ, since CQ has not actually been used as a monotherapy. Possible variations of total glutathione levels and GR and $\gamma$-GCS activities were determined in function of the therapeutic response in parasitized erythrocytes, non-infected erythrocytes and free parasites. It should help to understand the phenomena of therapeutic failure to the 4-aminoquinolines.

\section{Materials and methods}

Within the framework of the evaluation of the resistance to antimalarics by RAVREDA (Red Amazóniza de Vigilancia a la Resistencia de Drogas Antimaláricas), during 2003 and 2004, clinical and parasitological therapeutic response to monotherapy with AQ was assessed in patients with non-complicated $P$. falciparum malaria, according to the WHO Protocol 2000 that classifies the response in adequate clinical response (ACR) and therapeutic failure (TF). Patients were from Turbo and El Bagre, two townships in Antioquia, Colombia. In this study, the therapeutic failure was higher than permitted $(>26 \%)$ by National Health Ministry treatment guidelines in Colombia and, therefore, the study was carried out only with 32 patients. Initial malaria diagnosis was carried out with thick and thin smear; these were Field and Giemsa stained respectively. Parasitaemia was calculated by counting the number of asexual forms by 200 leucocytes [16]. Samples 
of venous blood with anticoagulant CPD were taken on the starting day (zero day, before ingestion of AQ) and failure day for those patients who failed (before the rescue treatment). Samples were kept in liquid nitrogen until their processing. Total glutathione concentration and the enzymatic activity of GR and $\gamma$-GCS were determined in infected erythrocytes, non-parasitized erythrocytes and free parasites.

\section{Ethical considerations}

A written informed consent from each patient was obtained and signed before being included in the research project. This study was approved by the Ethics Committee of the Facultad de Medicina of the Universidad de Antioquia.

\section{Sample thawing}

Blood samples were thawed according to the workshop protocol suggested in the course "Molecular Approaches to Malaria" - International Centre for Engineering and Biotechonology [17].

\section{Separation of parasitized and non-parasitized erythrocytes by Percoll gradients}

In thawed samples, parasitized and non-parasitized red blood cells were separated by Percoll gradients according to the methodology described by Omodeo-Sale et al 2003 with some modifications [18]. The thawed red blood cells pellet was taken to a haematocrit of $20-25 \%$ with serumfree RPMI and fractionated onto a Percoll $/ 4 \%$ sorbitol (wt/vol) gradient: (from bottom to top) 90, 80, 70, 60 and $40 \%$ percoll. Resuspended erythrocytes were over-layered on the gradient and centrifuged 2000 RPM at room temperature (RT) for $20 \mathrm{~min}$. Three layers were obtained: an upper layer which corresponds to parasitized red blood cells; an intermediate layer which contained a mix of parasitized and non-infected erythrocytes, and a bottom layer corresponding to non-parasitized erythrocytes. The upper layer was removed. To enrich the non-parasitized erythrocyte fraction, the other two lower layers were mixed and fractionated again in the same Percoll-sorbitol gradient. All layers were washed twice with fresh serum-free RPMI. $5 \mu \mathrm{l}$ from the upper layer and $2.5 \mu \mathrm{l}$ of the bottom layer were suspended in $200 \mu \mathrm{l}$ of distilled water.

\section{Separation of free parasites from parasitized red blood cells infected with $P$. falciparum}

The free parasites were obtained according to Hsiao protocol with some modifications [19]: the pellet of parasitized red blood cells obtained by separation with Percoll gradients was taken and the same volume of $0.1 \%$ saponin was added; it was mixed with vortex and then incubated during 10 minutes at $37^{\circ} \mathrm{C}$, by mixing gently. It was centrifuged at 2,000 rpm for 15 minutes at RT. The pellet obtained had the free parasites which were washed with buffer phosphate by centrifugation at 10,000 rpm during 5 minutes at RT until the red color disappeared. The pellet of free parasites was diluted in $200 \mu \mathrm{l}$ of distilled water.

\section{Enzymatic activity and quantification of total glutathione} The enzymatic activity of glutathione reductase (GR) and $\gamma$-glutamylcysteine synthetase ( $\gamma$-GCS), protein concentration and total glutathione content were determined in parasitized and non-parasitized erythrocytes and in free parasites, and were carried out in duplicate.

\section{Determination of glutathione reductase activity}

This was carried out according to the method described by Calberg I and Mannervik B 1985 [20]. Glutathione reductase enzymatic activity is based on the decay of the absorbance of NADPH at $340 \mathrm{~nm}$ measured during a 5-minute period at $30^{\circ} \mathrm{C}$.

\section{Determination of $\gamma$ glutamyl cysteine synthetase activity}

This was determined according to the method described by Estrada del Cueto et al. 1999 [21]. This technique assumes that enzymatic activity is equal to the change in NADH absorbance at $340 \mathrm{~nm}$ during 60 minutes at $37^{\circ} \mathrm{C}$. The absorbance obtained while adding pyruvate/kinase enzymatic mixture (PK/LDH Sigma-Aldrich) was taken as zero time; it was incubated and absorbance was read again.

\section{Determination of total glutathione concentration by HPLC}

The concentration of total glutathione (oxidized and reduced) was analysed as $\mathrm{mBBr}$ derivatives (thiolyte ${ }^{\circledast} \mathrm{Cal}-$ biochem) by reversed-phase HPLC. The method suggested by Luersen et al. 2000 was followed with some modifications [22]: to $20 \mu \mathrm{l}$ of sample (parasitized erythrocytes, non-infected erythrocytes or free parasites) in 1.5$\mathrm{ml}$ Eppendorf tubes placed on ice and protected from light were added $10 \mu \mathrm{l}$ of $\mathrm{NABH}_{4}$, in $0.066 \mathrm{M} \mathrm{NaOH}$ and $33 \%$ (v/v) dimethyl sulphoxide (DMSO), $6 \mu \mathrm{l}$ of $2 \mathrm{mM}$ EDTA and $1.65 \mathrm{mM}$ dithiothreitol, $6 \mu \mathrm{l}$ of octanol and 14 $\mu \mathrm{l}$ of $1.8 \mathrm{M} \mathrm{HCl}$. After three min $70 \mu \mathrm{l}$ of $1 \mathrm{M}$ ethylmorpholine buffer (pH 8.5), $134 \mu \mathrm{l}$ of deionized water and 14 $\mu \mathrm{l}$ of $5 \mathrm{mM}$ thiolyte ${ }^{\varpi}$ were added. The derivatization was carried out at $70^{\circ} \mathrm{C}$ for $10 \mathrm{~min}$ in the dark and was terminated by adding $26 \mu \mathrm{l}$ of $100 \%$ acetic acid. After $20 \mathrm{~min}$ on ice in the dark, the sample was extracted with $200 \mu \mathrm{l}$ of dichloromethane and centrifuged at 10,000 rpm at RT for two minutes. The supernatant, which is the water-soluble phase, was taken and filtered through a $0.45-\mu \mathrm{m}$ nylon membrane. The filtered samples were stored at $-20^{\circ} \mathrm{C}$, protected from light until their injection in the HPLC. 20 $\mu \mathrm{l}$ obtained from the water-soluble phase were injected on to a LiChroCART $^{\circledast} 100 \mathrm{RP}-18(5.0 \mu \mathrm{m})$ reverse phase HPLC. The column was eluted at a flow rate of $0.55 \mathrm{ml} /$ min by the following gradient of solvent A $(0.25 \%$ acetic 
acid) and solvent $\mathrm{B}$ (100\% acetonitrile): $0 \mathrm{~min}, 100 \%$ solvent A; $5 \mathrm{~min}, 90 \%$ solvent A; $20 \mathrm{~min}$, 85\% solvent A, 25 min, $0 \%$ solvent $A$. The effluent was monitored by a fluorescence spectrophotometer (excitation $400 \mathrm{~nm}$; emission $475 \mathrm{~nm}$ ). Under these conditions, the glutathione-thiolyte $^{\circledast}$ adduct had a retention time of $13.9 \mathrm{~min}$ and cysteine-thiolyte $^{\circledast}$ of $10.07 \mathrm{~min}$. Cysteine (Cys) and GSH were used as external standards with recovery rate of 123.8 $\pm 6.6 \%$ and $74.8 \pm 8.2 \%$ respectively. Two calibration curves were elaborated due to concentration variation of glutathione in the samples and in doing the regression analysis (lineal model). The obtained sensibility was 3.2 pmol, which is found in the quantity range detected when working derivatized compounds with monobromobimane [23].

\section{Determination of protein concentration}

Protein determination to relate enzymatic activity and the glutathione content was carried out by the method of Lowry et al 1951 [24].

\section{Statistical analysis}

The data were analysed by the one-way variance analysis (Mann-Whitney test) in order to compare mean of total glutathione concentration and GR and $\gamma$-GCS activities in parasitized red blood cells, non-parasitized and free parasites in function of the therapeutic response (ACR and TF). A matching test was applied (Wilcoxon test) to compare the means in both days (starting and failure day) in the group of patients that had therapeutic failure. A correlation analysis and a simple linear regression were made to assess the association between GR and $\gamma$-GCS activity in ACR and FT patients with parasitaemia, and glutathione concentration in all cellular compartments. A confidence level of $95 \%(\alpha=0.05$ o $p \leq 0.05)$ was applied to all estimates and statistical tests. The program SpSS 14 was used for the statistical analysis.

\section{Results}

The therapeutic response to monotherapy with AQ was assessed during 28 days in 32 patients with non-complicated malaria by $P$. falciparum; 21 patients came from the township of Turbo, and 11 from El Bagre. Mean age was 27 years $(8-60)$ old and 17 of them were male. The 32 patients evaluated were divided into two groups according to therapeutic response to AQ; 22 of them had ACR and $10 \mathrm{TF}(31.25 \%)$. On the starting day (zero day), mean parasitaemia was $8,699(880$ - 31,960) rings/ $\mu$ l for ACR patients and $9,944(580-38,400)$ rings/ $\mu$ l for TF patients; on the failure day, mean parasitaemia was 1,681 (26 $8,640)$ parasites/ $\mu$ l.

\section{Glutathione concentration and enzymatic activity in malaria patients before treatment (starting day)}

On the starting day for all patients, total glutathione and GR and $\gamma$-GCS activities between parasitized and non-parasitized erythrocytes were compared. Non-parasitized erythrocytes had higher total glutathione than the infected ones, both in ACR as well as FT patients (ACR $=16.324 \pm$ $4.992 \mathrm{nmol} / \mathrm{mg}$ protein vs $5.612 \pm 2.119 \mathrm{nmol} / \mathrm{mg}$ proteins, $p<0.001$ and $\mathrm{TF}=21.636 \pm 4.149 \mathrm{nmol} / \mathrm{mg}$ protein vs $11.725 \pm 4.833 \mathrm{nmol} / \mathrm{mg}$ proteins, $p=0.003)$. Also for both therapeutic response groups was found that GR activity was statistically lower in the healthy erythrocytes compared to parasitized erythrocytes $($ ACR $=23.691 \pm$ $8.145 \mathrm{UI} / \mathrm{mg}$ protein vs $34.927 \pm 14.777 \mathrm{UI} / \mathrm{mg}$ proteins, $p=0.015$ and $\mathrm{TF}=18.594 \pm 5.442 \mathrm{UI} / \mathrm{mg}$ protein vs $30.103 \pm 10.171 \mathrm{UI} / \mathrm{mg}$ proteins, $p=0.021)$. Differences were not found in the $\gamma$-GCS activity between both compartments for any of two groups of therapeutic response $(p>0.05)$ (Table 1$)$.

When the analysis was done based on the therapeutic response, we found that TF patients have higher total glutathione in parasitized erythrocytes and in non-parasitized erythrocytes compared with those that had ACR $(p<$ $0.016)$. There were no differences for GR and $\gamma$-GCS activities for either therapeutic response group (Table 1).

The glutathione content in free parasites was the same in all patients, sometimes it was below the method detection limit (3.2 pmol). Differences in GR and $\gamma$-GCS activities were not found for either therapeutic response group.

In correlation analysis and a simple linear regression, TF patients presented a 58\% correlation between parasitaemia and GR activity of free parasites, and statistically significant correlations were not found in any of the cellular compartments for ACR patients (always $\mathrm{r}^{2}<0.5$, data nonshown).

\section{Glutathione and enzymatic activity in FT patients}

Total glutathione and the activity of both enzymes were studied in the 10 TF patients. On the failure day, it was found that infected erythrocytes had lower total glutathione and higher GR y $\gamma$-GCS activities compared with nonparasitized erythrocytes $(p<0.014)$ (Table 1$)$. In the analysis of TF patients based on the day (starting and failure day), was seen a significant decrease in glutathione concentration of parasitized erythrocytes on failure day $(11.725 \pm 4.833$ vs $5.847 \pm 2.582, p=0.017)$ and there was no important changes in total glutathione of non-parasitized erythrocytes or in the GR and $\gamma$-GCS activity (Table 1). However, there was 93\% correlation between GR activity of parasitized erythrocytes and parasitaemia on the failure day. 


$\begin{array}{lllll}\text { ACR Starting day } & \text { Failure } & \text { ACR Starting day } & \text { Failure } & \text { ACR Starting day }\end{array}$

Starting day Failure day
$5.847 \pm 2.582^{c, j}$

$16.324 \pm 4.992^{\mathrm{a}, \mathrm{i}}$

$21.636 \pm 4.149 \mathrm{~b}$,

$19.093 \pm 8.288$

$2.416 \pm 0.073$

$8.700 \pm 5.867$

$41.901 \pm 17.110^{f}$

$23.691 \pm 8.145^{d}$

$18.594 \pm 5.442$

$21.999 \pm 8.903$
$34.927 \pm 14.777^{d}$

$30.103 \pm\left. 10.17\right|^{\mathrm{e}}$

$13.150 \pm 7.0688$
$10.043 \pm 1.549$

$9.850 \pm 1.50$
Starting day

Failure day

$\gamma$-GCS (mU/mg protein)

*In free parasites GR and $\gamma$-GCS enzymatic activity was not established because the protein quantity found was very low and in some cases not detectable, therefore the values shown correspond to enzymatic velocity $(\mathrm{mU} / \mathrm{ml})$.

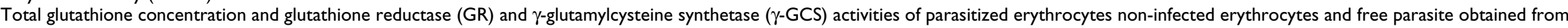

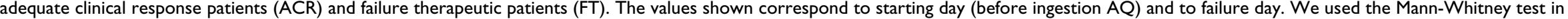

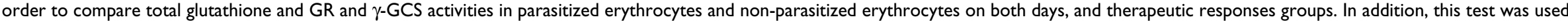

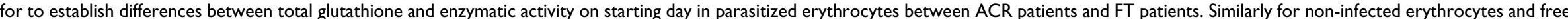

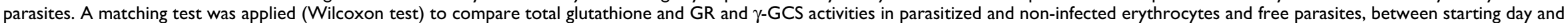
failure day in FT patients. $P$ values statistically significant are shown.

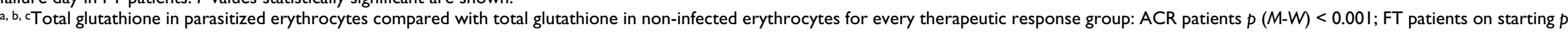
f $(M-W)=0.003$; and FT patients on failure day $p(M-W)=0.001$.

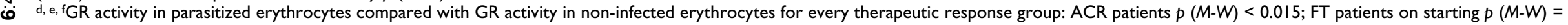
N 0.021 ; and FT patients on failure day $p(M-W)=0.013$.

gy-GCS activity in parasitized erythrocytes compared with $\gamma$-GCS activity in non-infected erythrocytes for every therapeutic response group: $\mathrm{FT}$ patients on failure day $p(M-W)=0.012$.

hTotal glutathione in parasitized erythrocytes of ACR patients compared with FT patients on starting day $p(M-W)=0.001$.

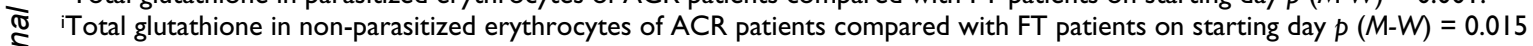

iTotal glutathione in parasitized erythrocytes of FT patients on starting day compared with FT patients on failure day $P(W$ Wilcoxon) $=0.017$ 


\section{Discussion}

In this study, AQ failure in patients with malaria by $P$. falciparum was $31.25 \%$, which is above the established value to stop a treatment schaeme. This is the reason why it was not possible to include a larger number of patients.

Luersen et al reported that non-parasitized erythrocytes from ring-synchronous cultures of $P$. falciparum have $44 \%$ more GSH than parasitized erythrocytes [22]. Although samples in this study come from malaria patients, this is in agreement with the results, where parasitized erythrocytes had lowered their total glutathione by more than $50 \%$. In non-infected erythrocytes, there is no Plasmodium-induced intra erythrocytic oxidative stress and glutathione efflux and oxidation. However, it is important to know if glutathione concentrations in this study correspond to its reduced state, in order to confirm the hypothesis formulated. The higher GR activity that was found on the starting day in the Plasmodium-infected erythrocytes could indicate that the contribution of the parasite to glutathione metabolism depends on its reduction rather than on its synthesis. GR activity found in free parasites supports this hypothesis. The $58 \%$ correlation between parasitaemia and GR activity in the free parasites in TF patients on starting day should be noted. This might explain a difference in glutathione metabolism not influenced by the presence of the drug and possibly favouring therapeutic failure.

Glutathione could compete with AQ for the haem group, possibly explaining therapeutic failure [7]. When glutathione content and the activity of both enzymes (GR y $\gamma$ GCS) in the different compartments studied are compared with therapeutic response on the starting day (before ingestion of AQ), we observed that parasitized and nonparasitized erythrocytes of FT patients had higher amount of total glutathione compared with ACR erythrocytes. This is in agreement with reports by Safeukui et al., who found that trophozoites of $P$. berghei CQ resistant strains isolated from infected and untreated mice have higher glutathione concentration in reduced form than in oxidized form, compared with trophozoites of $P$. berghei CQ sensitive and untreated [14]. The differences in parasitaemia do not explain the results we obtained because, on the starting day, parasitaemia is similar in both groups. A possible explication could be that the resistant parasites have a better redox system and, therefore, may have higher levels of total glutathione, resulting from the novo synthesis and reduction of oxidized glutathione. Differences in the enzymatic activity could not be observed in this study because, in the techniques used, NADH or NADPH could be consumed by other enzymes.

The detection limit of total glutathione was $3.2 \mathrm{pmol}$, which agrees with the report of Ivanov et al. that point out a detection limit of 2-5 pmol [23]. Nevertheless, the small amount of parasites possibly caused the total glutathione to be below the detection limit in free parasites and, therefore, could have been the cause for not finding differences.

Also, on failure day it was examined if there was a difference between the parasitized and non-parasitized erythrocytes in the total glutathione and enzymatic activities; a statistically higher total glutathione in non-infected erythrocytes compared with parasitized erythrocytes was found. These results agree with those found on the starting day and therefore could also be explained by the consumption of glutathione, product of the haemoglobin metabolism in the red blood cell infected with Plasmodium. In addition, was observed on failure day that GR as well as $\gamma$-GCS activities are statistically higher in the infected erythrocytes. It could be assumed that the maintenance of glutathione on the failure day depend on synthesis as well as reduction. This difference between starting and failure day possibly is due to the presence of the drug that increases the number of free radicals and, therefore, the parasite must also increase the glutathione novo synthesis in order to compensate the difference in reactive species that were controlled by greater GR activity on the starting day when the drug was not present.

When establishing the comparison between the starting day and failure day in TF patients at AQ, it was observed that, on the failure day, the total glutathione in parasitized erythrocytes was inferior to that of such patients in pretreatment starting day. As was mentioned above, it is possible that the presence of the drug increased the reactive species and thus increased the consumption of glutathione. This does not occur in the non-parasitized erythrocytes on the failure day, where the amount of total glutathione continues to be the same as on starting day. Nevertheless, these differences in the total glutathione of both days could also be attributed to the difference in parasitaemias, since they were lower on the failure day compared with starting day. Changes in enzymatic activities between the starting day and failure day were not observed. However, with the methodology used, was cannot discard that some difference exists.

With the results obtained, was concluded that:

1) Independent of the therapeutic response and of the treatment day (starting or failure), the total glutathione was higher and GR activity was lower in the non-infected erythrocytes compared with parasitized erythrocytes.

2) The higher GR activity in the infected erythrocytes on the failure day could be due to a compensation mechanism by lower total glutathione in this compartment. 
3) The patients with TF had higher total glutathione in parasitized and non-parasitized erythrocytes on the starting day compared with the ACR patients, indicating a possible contribution to the therapeutic failure.

4) On failure day, the maintenance of glutathione in parasitized erythrocytes depends on synthesis and reduction, possibly by the increase in the oxidative stress that AQ generates.

5) In the FT patients, the total glutathione found in infected erythrocytes was less on the failure day compared with starting day. This is possibly due to the larger amount of glutathione which is consumed in presence of the drug and also to lower parasitaemia.

This study is the first attempt to explain $P$. falciparum therapeutic failure through differences found in glutathione metabolism in FT and ACR patients. Our results may involve glutathione in the therapeutic response to antimalarials; nevertheless, further studies are needed to prove this relationship. It is also important to compare these results with samples of healthy individuals to rule out other possible differences. In addition, it is important to perform more accurate enzymatic assays to discard differences in the enzymatic activities between the treatment days and between the therapeutic response groups. It would be useful to do a follow-up of the glutathione during and after the malaric episode in patients with different therapeutic response to establish if the glutathion (GSH and GSSG) change during the infection and if they return to the same level when the parasite is not present.

\section{Authors' contributions}

LZ designed and managed the study, made the assays of separation cellular and evaluated enzymatic activity, analysed of data and wrote the first draft of the manuscript. AP designed study, analysed of data, contributed in the standardization of the techniques and cowrote the first draft of the manuscript. AO validated the method for quantification of total glutathione and made injection the samples to HPLC. CL coordinated the validated for quantification of total glutathione and analysed data. SB coordinated the study, analysed data and critically reviewed and suggested changes to the manuscript. All authors read and approved the final manuscript.

\section{Acknowledgements}

We acknowledge our research sponsors, CODI, Special Topic Research Funds 2003 of Universidad de Antioquia. We also thank the communities of Turbo and El Bagre townships for their collaboration. Thanks to Professor Silvio Ayala from the Facultad de Veterinaria y Zootecnia of the Universidad de Antioquia for lending us the HPLC in order to quantify the glutathione. Thanks to Professor Luis Carlos Burgos of the Universidad de Antioquia and Professor Juan Diego Maya of the Universidad de Chile, for their academic consulting. Thanks also to Gonzalo Álvarez for his advise on statistics and to Professor James Smith for his help with the translation.

\section{References}

I. Hoffman SL, Subramanian GM, Collins FH, Venter JC: Plasmodium, human and Anopheles genomics and malaria. Nature 2002, 415:702-709.

2. Stivanello E, Cavailler P, Cassano F, Omar SA, Kariuki D, Mwangi J, Piola P, Guthmann JP: Efficacy of chloroquine, sulphadoxinepyrimethamine and amodiaquine for treatment of uncomplicated Plasmodium falciparum malaria in Kajo Keji county, Sudan. Trop Med Int Health 2004, 9:975-80.

3. Blair S, Lacharme L, Carmona J: Resistance of Plasmodium falciparum to antimalarial drugs in Zaragoza (Antioquia, Colombia), 1998. Mem Inst Oswaldo Cruz 2002, 97:40I-406.

4. Blair S, Carmona-Fonseca J, Pineros JG, Rios A, Alvarez T, Alvarez G, Tobon A: Therapeutic efficacy test in malaria falciparum in Antioquia, Colombia. Malar J 2006, 5:14.

5. Sullivan $\mathrm{DJ}$ : Theories on malarial pigment formation and quinoline action. Int J Parasitol 2002, 32: I645-1653.

6. Ginsburg H, Famin O, Zhang J, Krugliak M: Inhibition of glutathione-dependent degradation of haeme by chloroquine and amodiaquine as a possible basis for their antimalarial mode of action. Biochaem Pharmacol 1998, 56: I305-1313.

7. Ginsburg H, Golenser J: Glutathione is involved in the antimalarial action of chloroquine and its modulation affects drug sensitivity of human and murine species of Plasmodium. Redox Rep 2003, 8:276-279.

8. Ginsburg H: Redox metabolism in malaria: from genes, through biochaemistry and pathology, to drugs. Redox Rep 2003, 8:23I-233.

9. Muller S: Redox and antioxidant systems of the malaria parasite Plasmodium falciparum. Mol Microbiol 2004, 53:I29|-I 305.

10. Becker K, Tilley L, Vennerstrom JL, Roberts D, Rogerson S, Ginsburg $\mathrm{H}$ : Oxidative stress in malaria parasite-infected erythrocytes: host-parasite interactions. Int J Parasitol 2004, 34:163-189.

II. Meierjohann S, Walter RD, Muller S: Regulation of intracellular glutathione levels in erythrocytes infected with chloroquinesensitive and chloroquine-resistant Plasmodium falciparum. Biochaem J 2002, 368:761-768.

12. Platel DF, Mangou F, Tribouley-Duret J: Role of glutathione in the detoxification of ferriprotoporphyrin IX in chloroquine resistant Plasmodium berghei. Mol Biochaem Parasitol 1999, 98:215-223.

13. Perez-Rosado J, Gervais GW, Ferrer-Rodriguez I, Peters W, Serrano AE: Plasmodium berghei: analysis of the gamma-glutamylcysteine synthetase gene in drug-resistant lines. Exp Parasitol 2002, 101:175-182.

14. Safeukui I, Mangou F, Malvy D, Vincendeau P, Mossalayi D, Haumont G, Vatan R, Olliaro P, Millet P: Plasmodium berghei: dehydroepiandrosterone sulfate reverses chloroquino-resistance in experimental malaria infection; correlation with glucose 6phosphate dehydrogenase and glutathione synthesis pathway. Biochem Pharmacol 2004, 68:1903-1910.

15. Deharo E, Barkan D, Krugliak M, Golenser J, Ginsburg H: Potentiation of the antimalarial action of chloroquine in rodent malaria by drugs known to reduce cellular glutathione levels. Biochem Pharmacol 2003, 66:809-817.

16. López-Antuñano F: Diagnóstico microscópico de los parásitos de la malaria en la sangre. Diagnóstico de malaria. Washington | 988,5 | 2:39-50.

17. International Center for Engineering and Biotechonology (ICGEB): Workshop Handbook. Molecular Approaches to malaria. New Delhi 2000.

18. Omodeo-Sale F, Motti A, Basilico N, Parapini S, Olliaro P, Taramelli $D$ : Accelerated senescence of human erythrocytes cultured with Plasmodium falciparum. Blood 2003, I02:705-7II.

19. Hsiao LL, Howard RJ, Aikawa M, Taraschi TF: Modification of host cell membrane lipid composition by the intra-erythrocytic human malaria parasite Plasmodium falciparum. Biochaem J 1991, 274:121-132.

20. Calberg I, Mannervik B: Glutathione Reductase. Methods in enzimology 1 985, I I 3:484-90.

21. Estrada del Cueto M, Molina G, Gutiérrez A, Pérez G: Enzimas de la biosíntesis del glutatión reducido (GSH). Valores nor- 
males y estudio de un paciente con deficiencia de GSH. Rev Cubana Haematol Haemotr 1999, 15:35-41.

22. Luersen K, Walter RD, Muller S: Plasmodium falciparum-infected red blood cells depend on a functional glutathione de novo synthesis attributable to an enhanced loss of glutathione. Biochaem J 2000, 346:545-552.

23. Ivanov AR, Nazimov IV, Baratova LA: Qualitative and quantitative determination of biologically active low-molecular-mass thiols in human blood by reversed-phase high-performance liquid chromatography with photometry and fluorescence detection. J Chromatogr A 2000, 870:433-442.

24. Lowry OH, Rosebrough NJ, Farr AL, Randall RJ: Protein measurement with the Folin phenol reagent. J Biol Chaem 195I, 193:265-75.

Publish with Bio Med Central and every scientist can read your work free of charge

"BioMed Central will be the most significant development for disseminating the results of biomedical research in our lifetime. "

Sir Paul Nurse, Cancer Research UK

Your research papers will be:

- available free of charge to the entire biomedical community

- peer reviewed and published immediately upon acceptance

- cited in PubMed and archived on PubMed Central

- yours - you keep the copyright

Submit your manuscript here:

http://www.biomedcentral.com/info/publishing_adv.asp 